hep-ph/0008082

DESY 00-113

\title{
Sfermion production at photon colliders
}

\author{
M. Klasen \\ II. Institut für Theoretische Physik, Universität Hamburg, \\ Luruper Chaussee 149, D-22761 Hamburg, Germany \\ E-mail: michael.klasen@desy.de
}

\begin{abstract}
We calculate total and differential cross sections for sfermion production in $e^{+} e^{-}$ annihilation and in photon-photon collisions with arbitrary photon polarization. The total cross section at a polarized photon collider is shown to be larger than the $e^{+} e^{-}$annihilation cross section up to the kinematic limit of the photon collider.
\end{abstract}

Key words: Supersymmetry, Sfermion, Polarization, Photon, Collider PACS: 12.38.Bx, 13.85.Qk, 13.88.+e, 14.80.Ly

Supersymmetry (SUSY) is an attractive extension of the Standard Model (SM) of particle physics. If it exists at the electroweak-scale, some or all of the SUSY partners of the SM particles will be discovered at the energies available at the next generation of hadron colliders, i.e. at Run II of the Fermilab Tevatron or at the CERN LHC. However, due to the large hadronic backgrounds, these colliders will not be able to measure the properties of the sparticles in detail. This will be the task of a future linear $e^{+} e^{-}$collider or a photon collider, where high-energy laser photons are backscattered from the incident lepton beams. In this paper we summarize a recent comparative analysis of sfermion production in $e^{+} e^{-}$annihilation and photon-photon collisions with arbitrary photon polarization [1].'

The inclusive cross section for photoproduction of sfermions in $e^{+} e^{-}$collisions

$$
\sigma_{e^{+} e^{-}}^{B}(S)=\int \mathrm{d} x_{1} f_{i / e}\left(x_{1}\right) \mathrm{d} x_{2} f_{j / e}\left(x_{2}\right) \mathrm{d} t_{\tilde{f}} \mathrm{~d} u_{\tilde{f}} \frac{\mathrm{d}^{2} \sigma_{i j}^{B}(s)}{\mathrm{d} t_{\tilde{f}} \mathrm{~d} u_{\tilde{f}}}
$$

$\overline{1}$ A FORTRAN program to generate total or differential cross sections for any sfermion type in polarized or unpolarized photon-photon collisions or in $e^{+} e^{-}$annihilation is available from the author upon request. 
can be obtained by convolving the hard photonic cross section

$$
\frac{\mathrm{d}^{2} \sigma_{i j}^{B}(s)}{\mathrm{d} t_{\tilde{f}} \mathrm{~d} u_{\tilde{f}}}=\frac{1}{16 \pi s^{2}} \Theta\left(t_{\tilde{f}} u_{\tilde{f}}-m_{\tilde{f}}^{2} s\right) \Theta\left(s-4 m_{\tilde{f}}^{2}\right) \delta\left(s+t_{\tilde{f}}+u_{\tilde{f}}\right){\overline{\left|M_{i j}^{B}\right|}}^{2}
$$

with the energy spectrum for backscattered laser photons $f_{\gamma / e}(x)[2]$. In addition to the direct contributions with $i, j=\gamma$, one or two of the photons can also resolve into a hadronic structure before they interact. However, these contributions are numerically only significant for small, experimentally excluded, squark masses [1].

$$
\begin{aligned}
{\overline{\left|M_{\gamma \gamma}^{B}\right|}}^{2} & =\frac{4 e^{4} e_{\tilde{f}}^{4} N_{C}}{t_{\tilde{f}}^{2} u_{\tilde{f}}^{2}}\left\{t_{\tilde{f}}^{2} u_{\tilde{f}}^{2}\left[1+\tilde{\xi}_{1}^{(1)} \tilde{\xi}_{1}^{(2)}-\tilde{\xi}_{2}^{(1)} \tilde{\xi}_{2}^{(2)}+\tilde{\xi}_{3}^{(1)} \tilde{\xi}_{3}^{(2)}\right]\right. \\
& -2 m_{\tilde{f}}^{2} t_{\tilde{f}} u_{\tilde{f}} s\left[\tilde{\xi}_{1}^{(1)} \tilde{\xi}_{1}^{(2)}-\tilde{\xi}_{2}^{(1)} \tilde{\xi}_{2}^{(2)}+\left(1+\tilde{\xi}_{3}^{(1)}\right)\left(1+\tilde{\xi}_{3}^{(2)}\right)\right] \\
& \left.+2 m_{\tilde{f}}^{4} s^{2}\left(1+\tilde{\xi}_{3}^{(1)}\right)\left(1+\tilde{\xi}_{3}^{(2)}\right)\right\}
\end{aligned}
$$

is the photonic matrix element squared, which has been calculated using a covariant density matrix for polarized photons [1]. The polarization states of the incoming photons with momenta $k_{1,2}=\sqrt{s} / 2(1,0,0, \pm 1)$ are determined by the Stokes parameters $\tilde{\xi}_{i}, i=1,2,3$, where $\sqrt{\tilde{\xi}_{1}^{2}+\tilde{\xi}_{3}^{2}}$ is the degree of linear polarization and

$$
\tilde{\xi}_{2}=\Delta f_{\gamma / e}(x) / f_{\gamma / e}(x) \quad, \quad \Delta f_{\gamma / e}(x)=f_{\gamma / e}^{+}(x)-f_{\gamma / e}^{-}(x)
$$

is the mean helicity. Since $\tilde{\xi}_{1}$ and $\tilde{\xi}_{3}$ depend on the azimuthal angle, cross sections with longitudinal photon polarization are difficult to disentangle and remain small if averaged over the azimuthal angle. Therefore we restrict ourselves to circularly polarized photons and set $\tilde{\xi}_{1}=\tilde{\xi}_{3}=0$. Since ${\overline{\left|M_{\gamma \gamma}^{B}\right|}}^{2}$ depends then only the product $\tilde{\xi}_{2}^{(1)} \tilde{\xi}_{2}^{(2)}$, we expect identical cross sections for incoming photons with identical or opposite helicities.

Summing over left- and right-handed squarks and sleptons has led to an additional factor of 2 in Eq. (3). The color factor $N_{C}=3$ for squarks, and $N_{C}=1$ for sleptons. It is important to note that the direct photon-photon cross section is proportional to the fourth power of the sfermion charge $e e_{\tilde{f}}$ $\left(e_{\tilde{u}}=2 / 3, e_{\tilde{d}}=-1 / 3, e_{\tilde{\ell}}=-1\right)$, to which it is very sensitive. The cross section is independent of the SUSY breaking mechanism, since it depends only on the physical sfermion masses $m_{\tilde{f}}$. The momenta of the outgoing sfermions are given by

$$
p_{1,2}=\left(m_{T} \cosh y, \pm p_{T} \cos \phi, \pm p_{T} \sin \phi, \pm m_{T} \sinh y\right)
$$




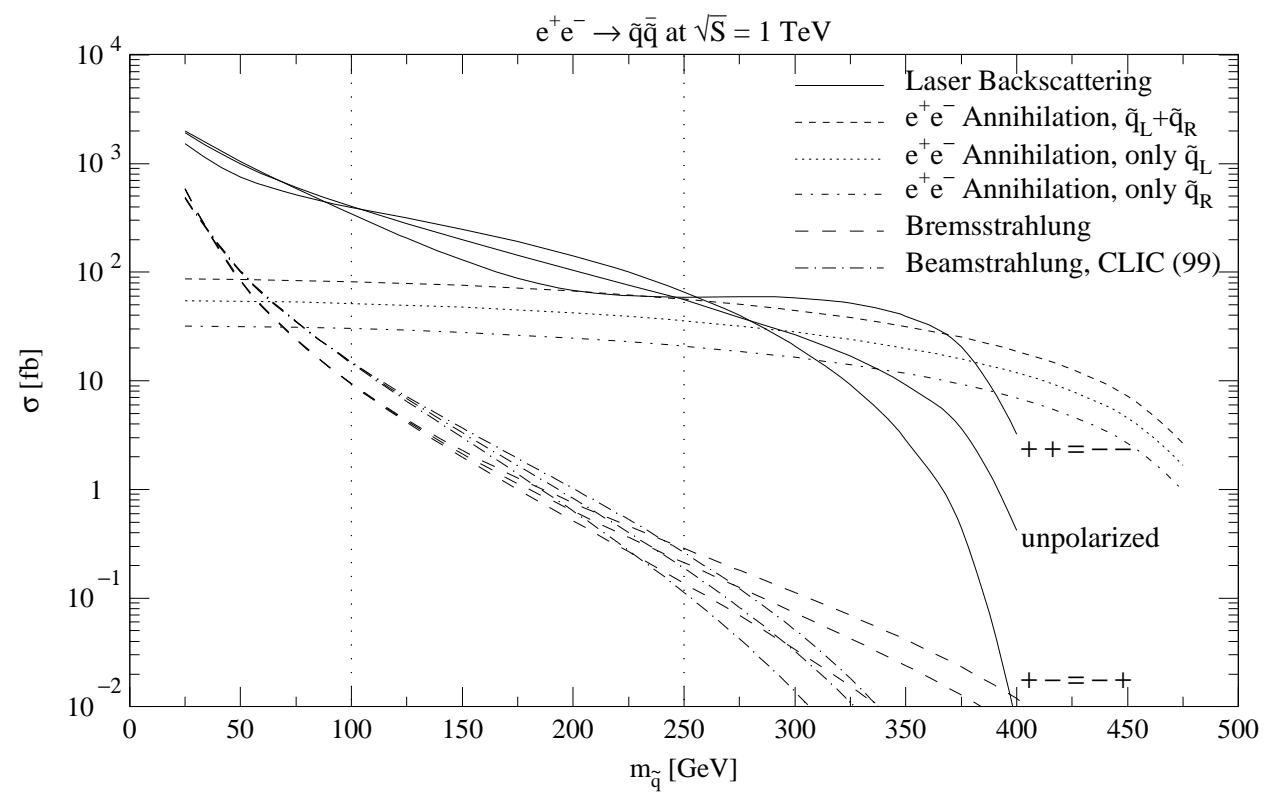

Fig. 1. Total cross section for up-type squark $\left(\tilde{q}_{L}+\tilde{q}_{R}\right)$ production in polarized photon-photon scattering and in $e^{+} e^{-}$annihilation at a $1 \mathrm{TeV}$ collider as a function of the squark mass $m_{\tilde{q}}$.

where $p_{T}, y$, and $\phi$ are the transverse momentum, rapidity and azimuthal angle of the produced sfermions, and $m_{T}=\sqrt{m_{\tilde{f}}^{2}+p_{T}^{2}}$ is the transverse sfermion mass. $s=\left(k_{1}+k_{2}\right)^{2}=x_{1} x_{2} S, t_{\tilde{f}}=\left(k_{2}-p_{2}\right)^{2}-m_{\tilde{f}}^{2}$, and $u_{\tilde{f}}=\left(k_{1}-p_{2}\right)^{2}-m_{\tilde{f}}^{2}$ are the Mandelstam variables of the hard photon-photon scattering process.

In Fig. 1 we compare the $e^{+} e^{-}$annihilation cross section against the polarized photon-photon cross section for up-type squark production at a $1 \mathrm{TeV}$ photon collider as a function of the squark mass. The labels,,++--+- , and -+ denote the helicities $P_{c}$ of the incoming laser photons. The helicities of the incoming leptons $\lambda_{e}$ have always been chosen to ensure the condition for optimal monochromaticity, $2 \lambda_{e} P_{c}=-1$. The backscattered photons are therefore highly polarized in the direction opposite to the laser photon [2]. Fig. 1 demonstrates that the unpolarized photon-photon cross section can be enhanced in the region $m_{\tilde{f}} \in[100 ; 250] \mathrm{GeV}$ by about $40 \%$ if one chooses opposite laser photon helicities $(+-$ or -+$)$. For $m_{\tilde{f}}>250 \mathrm{GeV}$ the effect is even more dramatic: The cross section can be improved by almost an order of magnitude at large $m_{\tilde{f}}$ if one chooses identical laser photon helicities. The cross section at a polarized photon collider stays larger than the $e^{+} e^{-}$ annihilation cross section almost up to the kinematic limit of the photon collider. It is interesting to note that one has to switch from opposite to identical helicities at $m_{\tilde{f}} \simeq 250 \mathrm{GeV}$, where the unpolarized photon-photon cross section drops below the annihilation cross section. While up-squarks below 250 


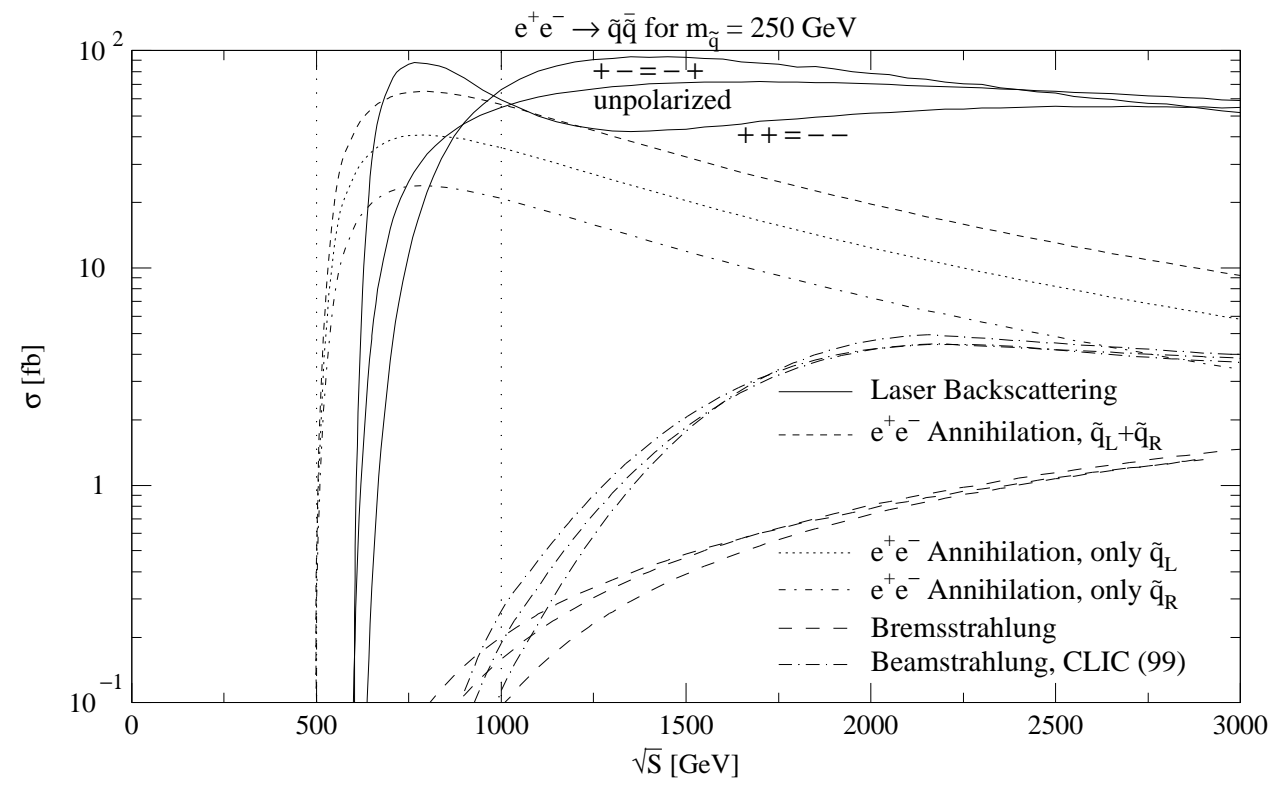

Fig. 2. Total cross section for the production of up-type squarks $\left(\tilde{q}_{L}+\tilde{q}_{R}\right)$ of mass $250 \mathrm{GeV}$ in polarized photon-photon scattering and in $e^{+} e^{-}$annihilation as a function of the center-of-mass energy $\sqrt{S}$.

$\mathrm{GeV}$ are already excluded by current Tevatron data, light stops and sleptons with $m_{\tilde{t}_{1}, \tilde{\ell}}>100 \mathrm{GeV}$ are still allowed. Note that the photon cross section for light left- (right-) handed sfermions has to be divided by 2 and compared to the left- (right-) handed annihilation cross section. Slepton cross sections are larger than up-type squark cross sections by a factor $1 /\left(3 e_{\tilde{u}}^{4}\right)=27 / 16$, while down-squark cross sections are smaller by a factor $\left(e_{\tilde{d}} / e_{\tilde{u}}\right)^{4}=1 / 16$. Selectron production in $e^{+} e^{-}$annihilation is enhanced by the $t$-channel exchange of a neutralino. In Fig. 1 we also show polarization effects for sfermions produced via brems- and beamstrahlung. The cross sections remain small and are only slightly enhanced by preferring identical over opposite lepton helicities.

In Fig. 2 we compare the same cross sections for a fixed up-type squark mass of $250 \mathrm{GeV}$ as a function of the center-of-mass energy of the collider $\sqrt{S}$. The unpolarized photon cross section can again be enhanced by an appropriate choice of polarization. In particular, identical laser photon helicities lead to a photon cross section which is larger than the annihilation cross section already at the threshold of the photon collider, i.e. below $1 \mathrm{TeV}$. Polarization for brems- and beamstrahlung is again of little interest. At large $\sqrt{S}$, where the cross sections become large, the radiated photons are completely unpolarized.

For experimental analyses it is also important to study differential cross sections, e.g. in the transverse momentum $p_{T}$ or the rapidity $y$ of the produced 


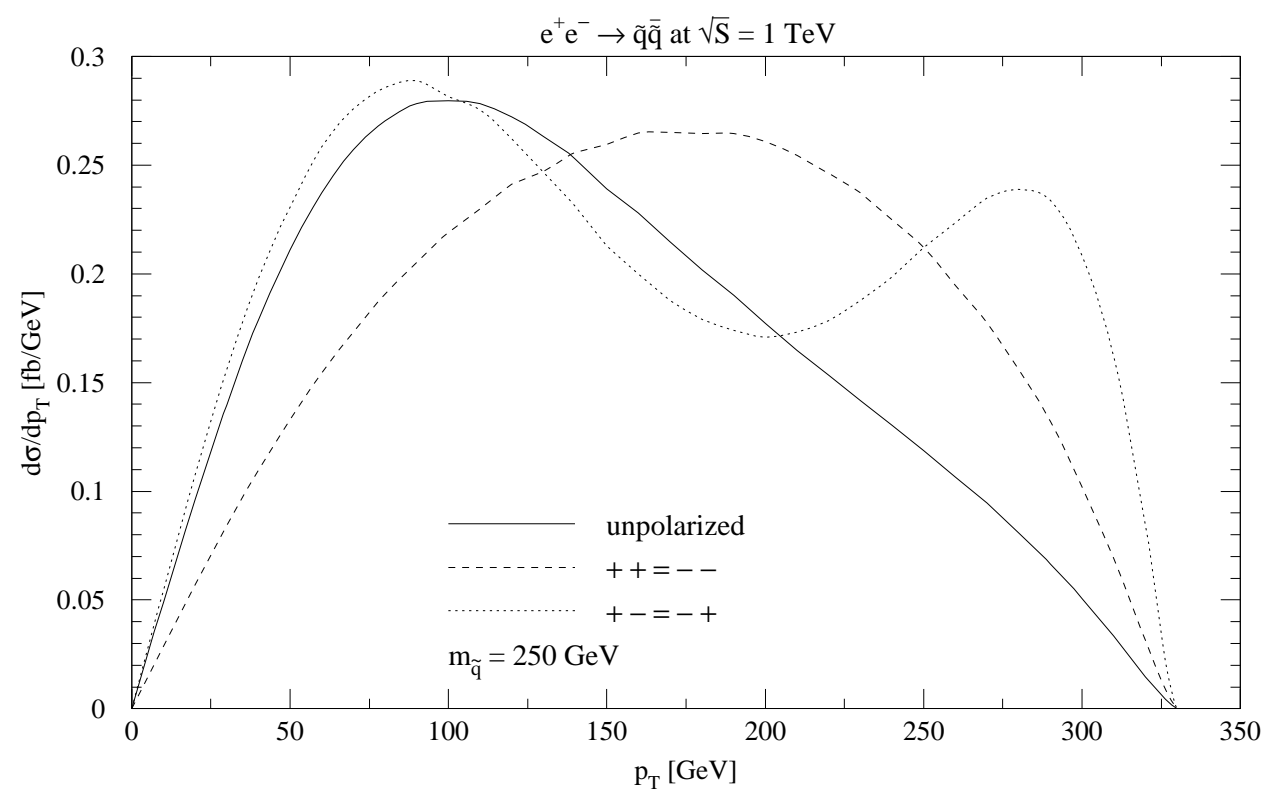

Fig. 3. Differential cross section for the production of up-type squarks $\left(\tilde{q}_{L}+\tilde{q}_{R}\right)$ of mass $250 \mathrm{GeV}$ at a $1 \mathrm{TeV}$ polarized photon collider as a function of the transverse momentum $p_{T}$.

supersymmetric particles

$$
\frac{\mathrm{d} \sigma_{e^{+} e^{-}}^{B}(S)}{\mathrm{d} p_{T} \mathrm{~d} y}=2 p_{T} S \int \mathrm{d} x_{1} x_{1} f_{i / e}\left(x_{1}\right) \mathrm{d} x_{2} x_{2} f_{j / e}\left(x_{2}\right) \frac{\mathrm{d}^{2} \sigma_{i j}^{B}(s)}{\mathrm{d} t_{\tilde{f}} \mathrm{~d} u_{\tilde{f}}}
$$

since cuts on $p_{T}$ and $y$ can help to eliminate backgrounds. For this reason we show in Fig. 3 differential $p_{T}$ spectra for up-type squarks of mass $250 \mathrm{GeV}$, produced at a $1 \mathrm{TeV}$ photon collider. The spectra have been integrated over the rapidity $y$ and extend out to the kinematic limit $p_{T}<0.828 \sqrt{S}-2 m_{\tilde{f}}=328$ $\mathrm{GeV}$. While the unpolarized spectrum peaks at $p_{T} \simeq 100 \mathrm{GeV}$, or roughly at $m_{\tilde{f}} / 2$, the mean $p_{T}$ of sfermions produced with photons of identical helicity is almost twice as big. If the photons have opposite helicity, one gets a distinct twin-peak behavior. This is due to the absence of a four-point interaction diagram which contributes only for identical helicities at intermediate $p_{T}$. These features should be very helpful in experimental analyses.

Finally we show in Fig. 4 rapidity distributions for up-type squarks of mass 100 $\mathrm{GeV}$ produced at a $1 \mathrm{TeV}$ photon collider. The rapidity spectra are symmetric around $y=0$ and extend out to $|y|<2$. This range should be covered by the detector at a photon collider to provide optimal analyzing conditions for sfermions of mass $m_{\tilde{f}}=100 \mathrm{GeV}$. For $m_{\tilde{f}}=250 \mathrm{GeV}$ the rapidity spectrum is narrower and extends out to $|y|<1$.1. The spectrum for laser photons 


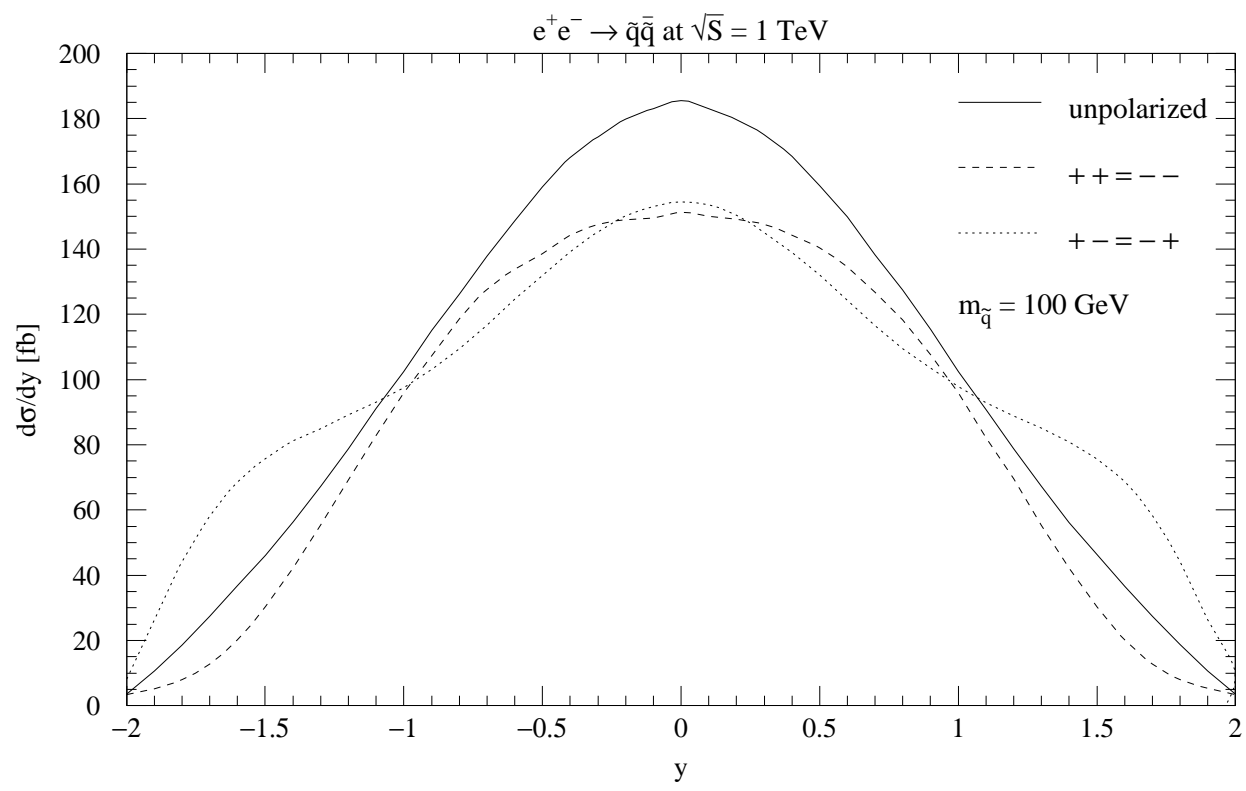

Fig. 4. Differential cross section for the production of up-type squarks $\left(\tilde{q}_{L}+\tilde{q}_{R}\right)$ of mass $100 \mathrm{GeV}$ at a $1 \mathrm{TeV}$ polarized photon collider as a function of the rapidity $y$. with identical helicities is again very similar to the unpolarized spectrum. The spectrum for opposite helicities has interesting shoulders at $y= \pm 1.5$. The dips at $y= \pm 1$ are again due to the absence of the four-point interaction diagram for opposite helicities.

We conclude that a photon collider may be advantageous for the analysis of sfermions for several reasons: Sfermion photoproduction is a pure SUSY-QED process and thus independent of a particular SUSY breaking mechanism. The cross section is very sensitive to the sfermion charge, and the polarized cross section is larger than the $e^{+} e^{-}$annihilation cross section up to the kinematic limit of the photon collider.

Acknowledgments. The author thanks the organizers for the kind invitation, S. Berge and Y. Umeda for their collaboration, and the Deutsche Forschungsgemeinschaft for financial support through Grant No. KL 1266/1-1.

\section{References}

[1] S. Berge, M. Klasen, Y. Umeda, DESY 00-112, hep-ph/0008081.

[2] I. F. Ginzburg, G. L. Kotkin, S. L. Panfil, V. G. Serbo and V. I. Telnov, Nucl. Instrum. Meth. A219 (1984) 5; V. I. Telnov, Nucl. Instrum. Meth. A294 (1990) 72 . 